\title{
A nálisis comparativo de indicadores en los estudios de utilización de medicamentos
}

\author{
E. Pastor García, J. Ma . Eiros Bouza*, A. Mayo Iscar** \\ Médico de Familia. C.S. Rondilla I. Valladolid. *Departamento de M icrobiología. \\ Facultad de Medicina. Hospital Clínico. Valladolid. **Departamento de Bioestadísti - \\ ca. Facultad de Medicina. Valladolid.
}

\section{RESUMEN}

Fundamentos: los estudios de utilización de medicamentos (EUM) precisan de indicadores pre cisos y universalmente aceptados para poder esta blecer comparaciones de sus resultados entre re giones y/o países así como a lo largo del tiempo en una zona determinada.

Objetivo: comparar dos indicadores usados am pliamente en los EUM: la dosis diaria definida (DDD) y las unidades de venta (envases).

Diseño: estudio longitudinal retrospectivo

Material y métodos: medimos el consumo de an tibióticos de uso sistémico en la provincia de Valla dolid en el periodo 1996-2000 a través de los datos proporcionados por IMS (International Marketing Services), estableciendo dicho consumo en DDD/1.000 habitantes/dia y en envases/1.000 ha bitantes/año.

Resultados: los datos obtenidos por los dos sis temas de indicadores son distintos, por un lado el estudio interanual en DDD muestra un incremen to en el uso de antibióticos entre 1996 y 1999, mientras que al medirlo en envases la curva obtenida es decreciente durante todo el periodo. Por otra parte la clasificación de los subgrupos de antibióticos por importancia de consumo se vio alterada.

Conclusiones: los resultados en los EUM se ven afectados en función de la unidad de medida elegi da. En nuestra opinión la DDD presenta notables ventajas respecto a la medida en envases.

Palabras clave: Estudios de utilización de medi camentos. Indicadores. Dosis diaria definida.
Comparative analysis of indicators in the studies of drug use

\section{ABSTRACT}

Backgrounds: studies of drug use (SDU) require precise and universally accepted indicators to be able to establish comparisons of their results among regions and/or countries as well as along the time in a certain area.

Objective: to compare two indicators widely used in SDU: the defined daily dose (DDD) and the sale units (packs).

Design: longitudinal retrospective study.

Material and methods: we measure the con sumption of antibiotics for systemic use in the county of Valladolid in the period 1996-2000 through the data provided by IMS (International Marketing Services), establishing this consumption in DDD/1,000 inhabitants/day and in packs/1,000 inhabitants/year.

Results: the data obtained by the two systems of indicators are different, on the one hand the intera nual study in DDD shows an increment in the use of antibiotics between 1996 and 1999, while when measuring it in packs the obtained curve is falling during the whole period. On the other hand the classification of the subgroups of antibiotics by im portance consumption was altered.

Conclusions: the results in the SDU are affected according to the measure unit elected. In our opi nion the DDD presents remarkable advantages re garding the measure in packs.

Key words: Studies of drug use. Indicators. De fined daily dose. 


\section{INTRODUCCIÓN}

Los estudios de utilización de medicamentos (EUM) forman parte de una disciplina de la farmacología conocida como farmacoepidemiología. Tienen como objetivo la mejora de la terapéutica farmacológica en el ámbito asistencial, residiendo su interés en cuatro puntos: determinar el coste de las necesidades farmacéuticas de la comunidad, analizar las posibles áreas de prescripción innecesaria, descubrir cualquier aumento de la morbilidad yatrogénica y formar una base sólida y fundada que permita supervisar la práctica de los profesionales de la atención de salud.

Según la OMS, los EUM comprenden el análisis de la comercialización, distribución, prescripción y uso de medicamentos en una sociedad, con acento especial sobre las consecuencias médicas, sociales y económicas resultantes. En esta definición no se incluyen los estudios controlados sobre eficacia (ensayos clínicos) ni los trabajos centrados específicamente en los efectos indeseables (farmacovigilancia) $)^{1,2}$.

Para llevar a cabo estos estudios son precisos unos indicadores adecuados que permitan realizar comparaciones de los resultados en el ámbito nacional e internacional. En este sentido el primer indicador usado fue el número de envases consumidos de una especialidad farmacéutica concreta, por ser una fuente de datos fácil de obtener y manejar para obtener un estudio estadístico. A partir de 1975 fue creado en Suecia el "Nordic Council on Medicines" que se planteó como primer objetivo la coordinación de cifras sobre el consumo de medicamentos en los países nórdicos, publicando las dosis diarias definidas (DDD) de los medicamentos registrados en Noruega.

El desarrollo del concepto de la DDD supuso un importante avance en la determinación de la unidad técnica ideal para el establecimiento de datos comparativos entre medicamentos. Su valor procura corresponderse con la dosis media diaria de mantenimiento en adultos de un medicamento para su indicación principal, por una vía de administración determinada, y normalmente se expresa en gramos de principio activo ${ }^{3-5}$. La OMS, a través del WHO Collaborating Centre for Drug Statis tics Methodology de Oslo (Noruega), las establece y revisa anualmente para un elevado número de fármacos ${ }^{4,6}$.

Actualmente la mayoría de los EUM utilizan como base la DDD, aunque diversos autores prefieren seguir usando como indicador el número de envases aduciendo diversas razones. En el presente trabajo realizamos un estudio comparativo de los dos tipos de indicadores señalados, analizando el consumo de antibióticos en la provincia de Valladolid durante el periodo 1996-2000.

\section{MATERIAL Y MÉTODOS}

Partimos del consumo de antibacterianos que se incluyen en la Anatomical Therapeutical Chemical Classification Index del grupo J01 (antibióticos de uso sistémico), no analizándose por tanto otros antiinfecciosos distintos de este grupo: antisépticos urinarios (G04A), antimicóticos de uso sistémico (J02), antimicobacterianos (J04), antivíricos de uso sistémico, sueros inmunológicos e inmunoglobulinas (J06) y vacunas (J07). Tampoco se incluyeron preparados tópicos dermatológicos, oftalmológicos o de la esfera ORL en cuya composición formen parte antibióticos de este grupo.

La información utilizada en el estudio procede de los datos auditados por IMS (International Mar keting Services), que analiza el consumo de fármacos en los países de nuestro entorno a partir de su salida desde el mayorista a las farmacias. La información de IMS no recoge el consumo hospitalario, pero puesto que prácticamente el $90 \%$ del consumo de antibióticos se realiza en Atención Primaria y que IMS sí contempla las ventas por prescripciones en consultas privadas y por automedicación, la muestra utilizada controla prácticamente el $95 \%$ del consumo global.

Los datos poblacionales se obtuvieron a partir del padrón municipal de 1996? .

\section{Descripción de los indicadores de consumo}

\section{Número de DDD por 1.000 habitantes y día (DHD)}

Por medio de la DDD, relacionamos la variable cualitativa principio activo (agrupando a todas las especialidades de composición común) con la variable cuantitativa Número de DDD por 1.000 habitantes y día $a^{3,5,89}$ (DHD), que proporciona una estimación de la prevalencia de consumo.

$$
\mathrm{DHD}=\frac{\mathrm{UV} \times \mathrm{FF} / \mathrm{E} \times \mathrm{C} / \mathrm{FF} \times 1.000}{\text { DDD } \times \mathrm{N}^{\circ} \text { de habitantes } \times 365 \text { días }}
$$

$\mathrm{UV}=$ unidades vendidas.

$\mathrm{FF} / \mathrm{E}=$ número de formas farmacéuticas por envase.

$\mathrm{C} / \mathrm{FF}=$ cantidad de producto en cada forma farmacéutica.

\section{Unidades de venta}

El indicador usado en este caso es el Número de envases por 1.000 habitantes y año (Env/1.000/año). 


$$
\text { Env/1.000/año }=\frac{\mathrm{N}^{\mathrm{o}} \text { de envases x } 1.000}{\mathrm{~N}^{\mathrm{o}} \text { de habitantes }}
$$

Para el procesamiento de los datos se construyeron bases de datos en el programa Excel y Access. El análisis de los datos se hizo utilizando el paquete estadístico SAS (North Caroline). Los resultados del análisis se presentan según los indicadores de consumo en tablas y figuras.

\section{RESULTADOS}

Hemos realizado dos tipos de análisis donde podemos valorar las diferencias observadas en función del indicador propuesto: en primer lugar valoramos el consumo interanual global de los antibióticos de uso sistémico y posteriormente estudiamos comparativamente los porcentajes de consumo de los distintos subgrupos de antibióticos en los cinco años conjuntamente.

La evolución del consumo de antibióticos de uso sistémico durante el quinquenio 1996-2000 está representada en las figuras 1 y 2 , pudiendo objetivar un desarrollo interanual diferente según el indicador escogido: en DHD dicho consumo presentó un curso fluctuante, con un aumento progresivo durante los cuatro primeros años, alcanzando las 23,24 DHD en 1999 para disminuir en el año 2000 hasta las 21,43 DHD (Fig. 1). Al tomar el envase como sistema de medida, pudimos observar una tendencia estable durante los cuatro primeros años, con cifras que oscilaron entre 1.688 y 1.747 envases por 1.000 habitantes y año, disminuyendo de forma importante durante el último año (1.532 envases/ 1.000 hab./año) (Fig. 2).

Cuando evaluamos el consumo por subgrupos de antibióticos objetivamos cambios sustanciales en los resultados en función del indicador elegido, de manera que tanto la clasificación por importancia de consumo como el porcentaje de cada subgrupo se modificaron ostensiblemente (Tablas I y II):

1. Las penicilinas de amplio espectro en ambos casos son los antibióticos más consumidos, pero mientras que en DHD suponen casi el 60\% del total, por envases no alcanzan el 50\%.

2. El subgrupo de macrólidos y lincosamidas se mantuvo en la segunda posición de máximo consumo en ambos casos, pero al contrario que en el subgrupo precedente el porcentaje es mayor al medirlo por envases que en DHD (21,34 frente a 16,36\%).

3 . Las quinolonas presentaron un consumo porcentual muy variable en función del indicador, siendo del 9,30\% en DHD (y tercer puesto por orden de consumo) y del 6,93\% en envases, situándose de esta forma en la cuarta posición.

4. Las cefalosporinas mostraron un comportamiento opuesto a las quinolonas, estando en la cuarta posición de consumo en DHD y tercera por envases.

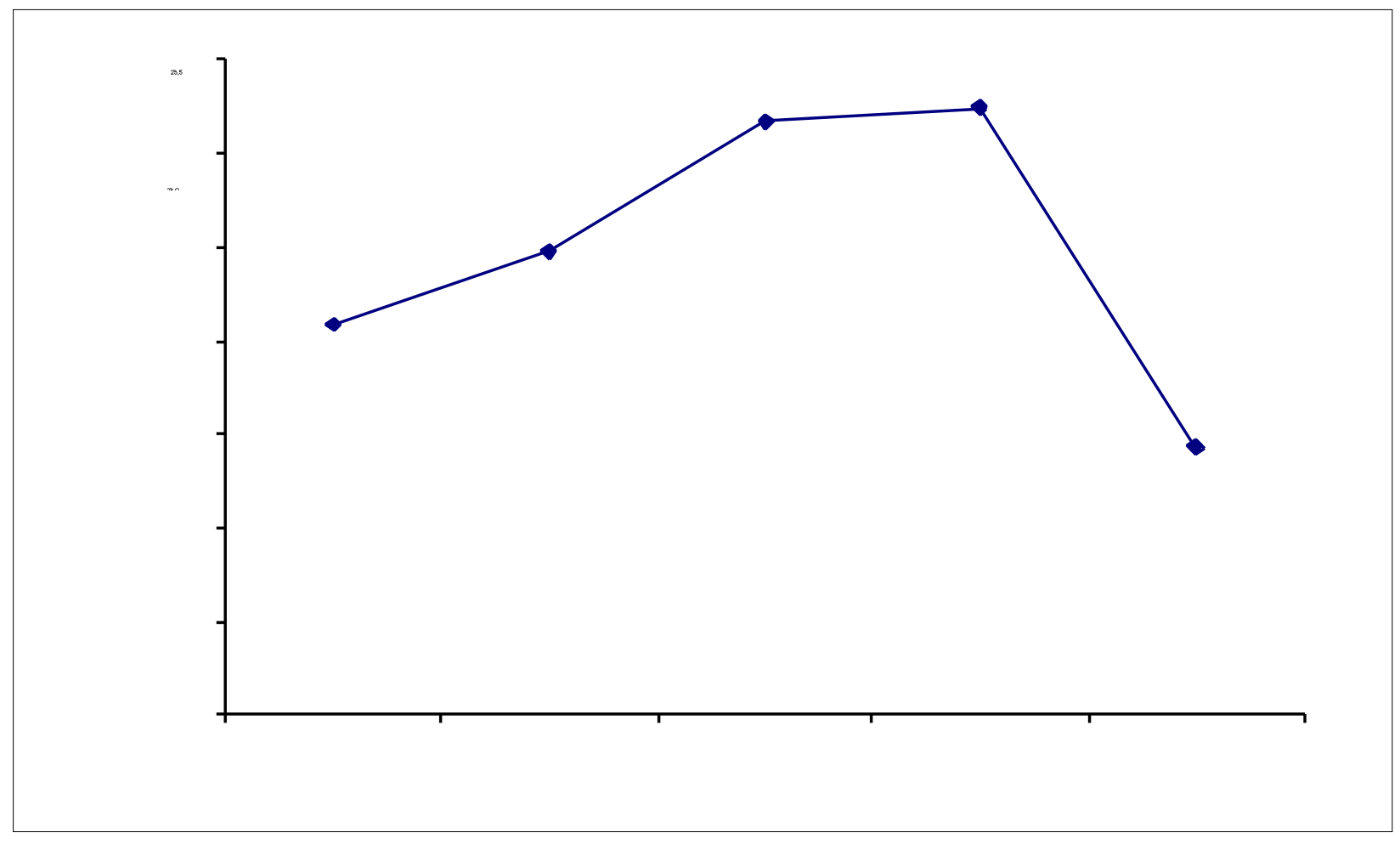

Figura 1

Evolución anual del consumo de antibióticos de uso sistémico en DDD/ 1.000 hab./ día en la provincia de Valladolid. 


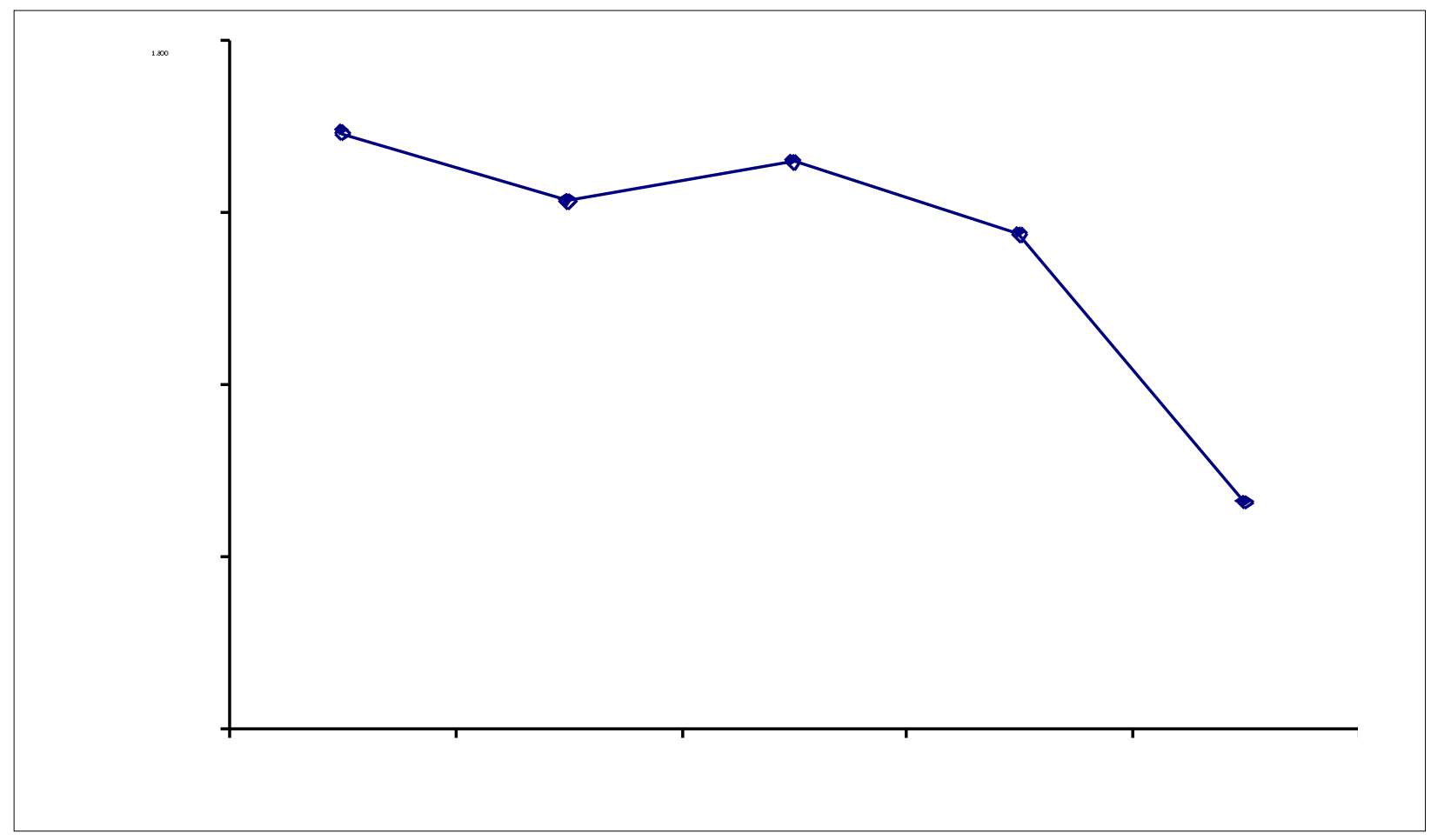

Figura 2

Evolución del número de envases de antibióticos de uso sistématico consumidos en la provincia Valladolid en función del año.

\begin{tabular}{|c|c|c|}
\hline \multicolumn{3}{|c|}{$\begin{array}{c}\text { CONSUMO MEDIO ANUAL DE LOS ANTIBIÓTICOS SISTÉMATICOS } \\
\text { CATEGORIZADOS POR SUBGRUPO TERAPÉUTICO EN DDD/1.000 } \\
\text { HAB./DÍA EN EL PERIODO 1996-2000 }\end{array}$} \\
\hline SUBGRUPO & MEDIA ANUAL & $\%$ \\
\hline $\begin{array}{l}\text { Penicilinas de amplio espectro } \\
\text { Macrólidos y lincosamidas } \\
\text { Quinolonas } \\
\text { Cefalosporinas } \\
\text { Tetraciclinas } \\
\text { Penicilinas resistentes a penicilinasa } \\
\text { Sulfonamidas y Trimetoprim } \\
\text { Otros antibióticos } \\
\text { Penicilinas sensibles a penicilinasa } \\
\text { Aminoglucósidos } \\
\text { Amfenicoles } \\
\text { Monobactams } \\
\text { Carbapenems }\end{array}$ & $\begin{array}{l}66,36 \\
18,38 \\
10,45 \\
9,10 \\
3,67 \\
1,96 \\
1,89 \\
0,44 \\
0,06 \\
0,04 \\
<0,01 \\
<0,01 \\
<0,01\end{array}$ & $\begin{array}{l}59,06 \\
16,36 \\
9,30 \\
8,10 \\
3,27 \\
1,74 \\
1,68 \\
0,39 \\
0,06 \\
0,04 \\
<0,01 \\
<0,01 \\
<0,01\end{array}$ \\
\hline TOTAL ANUAL & 112,36 & 100 \\
\hline
\end{tabular}

5. Las tetraciclinas fueron en ambos casos el quinto subgrupo más consumido con poca diferencia de resultados.

6. El resto de subgrupos presentaron consumos muy inferiores a los comentados previamente, aunque dentro de los resultados destacó la gran diferencia porcentual en el caso de las penicilinas sensibles a la penicilinasa, donde observamos que la

\begin{tabular}{|c|c|c|}
\hline \multicolumn{3}{|c|}{$\begin{array}{l}\text { CONSUM O MEDIO ANUAL DE ANTIBIÓTICOS SISTÉMATICOS } \\
\text { CATEGORIZADOS POR SUBGRUPO TERAPÉUTICO EN } \\
\text { ENVASES/1.000 HAB./AÑO EN EL PERIODO 1996-2000 }\end{array}$} \\
\hline SUBGRUPO & MEDIA ANUAL & $\%$ \\
\hline $\begin{array}{l}\text { Penicilinas de amplio espectro } \\
\text { Macrólidos y lincosamidas } \\
\text { Cefalosporinas } \\
\text { Quinolonas } \\
\text { Tetraciclinas } \\
\text { Penicilinas resistentes a penicilinasa } \\
\text { Sulfonamidas y Trimetoprim } \\
\text { Penicilinas sensibles a penicilinasa } \\
\text { Otros antibióticos } \\
\text { Aminoglucósidos } \\
\text { Monobactamas } \\
\text { Carbapenems } \\
\text { Amfenicoles }\end{array}$ & $\begin{array}{l}832,64 \\
358,60 \\
177,61 \\
116,48 \\
60,81 \\
44,96 \\
29,78 \\
28,33 \\
19,57 \\
10,01 \\
1,29 \\
0,35 \\
0,05\end{array}$ & $\begin{array}{l}49,55 \\
21,34 \\
10,57 \\
6,93 \\
3,62 \\
2,68 \\
1,77 \\
1,69 \\
1,16 \\
0,60 \\
0,08 \\
0,02 \\
<0,01\end{array}$ \\
\hline TOTAL ANUAL & 1680,47 & 100 \\
\hline
\end{tabular}

medida en DHD alcanza el 0,06\% del total, mientras que por envases se eleva al 1,69\%.

\section{DISCUSIÓN}

La necesidad de usar y mantener un sistema único de medida en los EUM es obvio en cuanto uno de sus principales objetivos es el análisis compara- 
tivo de consumo entre regiones y países o a lo largo del tiempo. El uso de la DDD supone un importante avance en este sentido por constituir una medida internacionalmente aceptada y avalada por la OMS. A pesar de esto todavía algunos autores utilizan indicadores de consumo farmacoeconómicos (número de envases y precio por envase $)^{10,11-13}$ en lugar de los indicadores farmacoterapéuticos (DDD) aduciendo diversas razones:

1. La DDD se refiere a dosis de mantenimiento en adultos y puede subestimar el número total de personas en tratamiento con antibióticos dada la considerable cantidad de uso de estos en pediatría.

2. Existen dificultades a la hora de comparar unos estudios con otros por diversos motivos: el uso de DDD para amoxicilina $=1 \mathrm{~g}^{14}$ y $1,5 \mathrm{~g}^{15}$, el uso de dosis reales superiores a la DDD (más acorde con la práctica clínica), el desconocimiento del proceso patológico para el que se prescribe ${ }^{13,16}$, la distinta duración del tratamiento para cada enfermedad $^{13}$, así como el empleo de distinta metodología en los estudios.

La primera consecuencia con la que nos encontramos al cambiar la unidad de medida se estableció en la observación del consumo global a lo largo de todo el periodo del estudio, donde descubrimos en el estudio por envases una tendencia progresiva negativa, que contrasta con la evolución del consumo en DDD. Este proceso de disminución del número de envases con aumento de las dosis consumidas en un periodo de tiempo determinado ha sido ya observado por Ruiz Bremón et al. en un trabajo en el que realizaron estudios paralelos con varios indicadore ${ }^{17}$ pudiendo estar causado por diversos motivos:

1. El uso de nuevas formulaciones con mayor contenido en peso del fármaco activo en cada unidad de dosificación. En este sentido cabe citar como ejemplo la utilización de la amoxicilina-clavulánico con dosis de $875 \mathrm{mg}$ de amoxicilina, lo que supone un $75 \%$ más por unidad empleada respecto a la dosis más habitual de $500 \mathrm{mg}$.

2. El incremento en el número de unidades por envase a lo largo del estudio. Dentro del subgrupo de las penicilinas de amplio espectro es cada vez más frecuente la presentación de 24 unidades por envase sobre todo para la amoxicilina y la amoxicilina-clavulánico para realizar un tratamiento completo de 8 días cuando previamente eran precisos dos envases de 12 unidades.

3. La disminución de las formas inyectables, que por regla general, tienen bastantes menos unidades que las formas orales habiendo contribuido a la aparente disminución de consumo en varios estudios ${ }^{11,17}$.

Los resultados por subgrupos también dependieron de la unidad de medida, de forma que tanto la clasificación por importancia en el consumo como los porcentajes respecto al total son diferentes: en ambos casos las penicilinas de amplio espectro destacan en primer lugar, pero en DDD supusieron el $60 \%$ y en envases el $50 \%$. Para valorar este dato hay que tener en cuenta dos hechos: por una parte el valor de DDD para la amoxicilina (1 gramo), mientras que terapéuticamente se usa habitualmente 1,5 gramos al día; por otra parte se precisan en muchas ocasiones dos envases para un tratamiento antiinfeccioso completo. El primer supuesto producirá un aumento del resultado en DDD y lo segundo hará lo mismo con el resultado en envases.

En sentido contrario, en el subgrupo de macrólidos y lincosamidas los resultados de consumo fueron mayores al medirlos en DDD, debido sin duda a que en la mayoría de los tratamientos realizados con estos fármacos es suficiente un envase, con excepción de la espiramicina, cuyo máximo uso depende de su asociación con el metronidazol (Rhodogil@) y la eritromicina, para los cuales en muchas ocasiones es preciso realizar un tratamiento con dos envases.

El cambio de magnitud también alteró la prevalencia entre quinolonas y cefalosporinas, ocupando el tercer y cuarto puesto al medir el consumo en DDD y a la inversa cuando medimos dicho consumo por envases. Nuevamente es la diferencia de envases consumidos para realizar un tratamiento lo que marca esta disparidad: en muchas ocasiones son precisos dos envases del subgrupo de las cefalosporinas para realizar un tratamiento completo (lo cual aumenta al realizar tratamientos con cefonicida por ser la presentación de una dosis por envase), mientras que las quinolonas (y sus moléculas más usadas ciprofloxacino y norfloxacino) tienen presentaciones adecuadas para realizar un tratamiento con tan sólo un envase.

Por lo que respecta al resto de subgrupos y principios activos no encontramos comparativamente diferencias de consumo con un sistema u otro de medida, con excepción de las penicilinas sensibles a la penicilinasa, cuyo porcentaje de consumo es mucho mayor al medirlo por envases debido a que su uso es mayoritariamente por vía parenteral, con tan sólo una dosis por envase.

En nuestra opinión el estudio del consumo de fármacos por envases presenta menor sensibilidad que el llevado a cabo con la DDD como medida debido a las variaciones que presentan los envases, no sólo entre diferentes países, sino también en un mismo país a lo largo del tiempo, y en el caso concreto de los antibióticos nos encontramos con que las presentaciones que se pueden encontrar en el mercado de un mismo producto son muy diversas. Como ejemplo, la amoxicilina se encuentra en las farmacias en forma de cápsulas, comprimidos, suspensión, sobres y viales, con dosis de 250, 500, 750 y $1.000 \mathrm{mg}$ y presentaciones de 12,16 y 24 unidades, siendo el consumo de unas formas más prevalente que otras, con la consecuencia final de que el tratamiento estadístico de todas las unidades por igual produce un sesgo importante. 


\section{Bibliografía}

1. Laporte JR, Tognoni G. Estudios de utilización de medicamentos y farmacovigilancia. En: Laporte JR, Tognoni G, eds. Principios de epidemiología del medicamento. $2^{\mathrm{a}}$ ed. Barcelona: Ed. Científicas y Técnicas, S.A., 1993; 1-24.

2. Laporte JR, Capellà D. El consumo de medicamentos. Med Clin (Barc) 1987; 89: 244-6.

3. García Iñesta A. Estudios de utilización de medicamentos en España y análisis de la situación farmacoterapéutica. En: Estudios de utilización de medicamentos. Madrid: Ed. Servicio de Documentación y Publicaciones del INSALUD, 1989; $17-48$

4. Davey PG, Bax RP, Newey J, Reever D, Rutherford D, Slack $\mathrm{R}$, et al. Growth in the use of antibiotics in the community in England and Scotland in 1980-1993. BMJ 1996; 312: 613.

5. Capellà D, Laporte JR. Métodos aplicados en estudios descriptivos de utilización de medicamentos. En: Laporte JR, Tognoni G, eds. Principios de epidemiología del medicamento. $2^{\text {a }}$ ed. Barcelona: Masson-Salvat, 1993; 67-93.

6. WHO Collaborating Centre for Drug Statistics Methodology: Anatomical Therapeutical Chemical (ATC) Classification Index. Including defined daily dosis (DDDs) for plain substances. Oslo: WHO CCDSM, 1995.

7. Base de Datos Municipales de Castilla y León: http://www.jcyl.es/jcyl/ceh/dge/

8. García Ruiz AJ, Fajardo Martín E, González Correa JA, Pavía Molina J, Sánchez de la Cuesta F. Utilización de antibacterianos en atención primaria en 1992. Farm Clin 1994; 11(5): 393-8

9. Martín Arias LH. Estudios de utilización de medicamentos.
En: Carbajal A. Farmacoepidemiología. Ed. Universidad de Valladolid.

10. Alós JI, Carnicero M. Consumo de antibióticos y resistencia bacteriana a los antibióticos: "algo que te concierne". Med Clin (Barc) 1997; 109: 264-70.

11. Calvo Plaza MI, Moreno Úbeda R, Domínguez Rojas V, Prieto Prieto J. Cinco años de evolución del consumo extrahospitalario de betalactámicos en España (1993-1997). Rev Esp Quimioter 2000; 13 (4): 417-24.

12. Carrie AG, Metge CJ, Zhanel GG. Antibiotic use in a canadian province, 1995-1998. Ann Pharmacother 2000 Apr; 34(4):459-64.

13. Segú i Tolsà JL. Prescripción de antibacterianos en Cataluña. Farm Clin 1989; 7(8): 679-88.

14. Yáñez Gómez P. Grado de utilización de antiinfecciosos de acción sistémica en un área de salud rural de Asturias. Comparación de los años 1994 y 1995. Aten Primaria 1997; 19 (5): 243-9.

15. Llop JC. Evolución en la utilización de antibacterianos en Cataluña. Impacto de los nuevos fármacos comercializados. Aten Primaria 1997; 19 (5): 230-6.

16. Sin Autor. Antibacterial Agens. En: Martidale. The extra pharmacopoeia. 32 ${ }^{\text {nd }}$ ed. London: James Reynods, 1999; 79224.

17. Ruiz Bremón A, Ruíz-Tovar M, Pérez Gorricho B, Díaz de Torres P, López Rodríguez R. Non-hospital consumption of antibiotics in Spain: 1987-1997. J Antimicrob Chemother 2000 Mar; 45 (3): 395-400. 